\title{
Dark energy model interacting with dark matter and unparticle
}

\author{
Songbai Chen* and Jiliang Jingt \\ Institute of Physics and Department of Physics, \\ Hunan Normal University, Changsha, Hunan 410081, P. R. China \\ Key Laboratory of Low Dimensional Quantum Structures \\ and Quantum Control of Ministry of Education, \\ Hunan Normal University, Changsha, Hunan 410081, P. R. China
}

\begin{abstract}
We study dynamical behaviors of the dark energy models interacting with dark matter and unparticle in the standard flat FRW cosmology. We considered four different interacting models and examined the stability of the critical points. We find that there exist late-time scaling attractors corresponding to an accelerating Universe and the alleviation of the coincidence problem depends on the choice of parameters in the models.
\end{abstract}

PACS numbers: 98.80.Cq, 98.80.-k

*Electronic address: csb3752@163.com

${ }^{\dagger}$ Electronic address: jljing@hunnu.edu.cn 


\section{INTRODUCTION}

Many observations confirm that we live in an accelerating Universe. Within the framework of Einstein gravity, this acceleration can be explained by the mysterious energy component named dark energy (DE) with negative pressure, which occupies almost $70 \%$ of the content of the universe at present [1, 2, 3], 4]. The simplest explanation for dark energy is the cosmological constant, which is a term that can be added to Einstein's equations. This term acts like a perfect fluid with an equation of state $(\mathrm{EoS}) \omega_{x}=-1$, and the energy density is associated with quantum vacuum. Although this interpretation is consistent with observational data, at the fundamental level it fails to be convincing. The vacuum energy density is far below the value predicted by any sensible quantum field theory, and it suffers the coincidence problem, namely, why the DE and the Dark Matter (DM) are comparable in size exactly right now. To overcome the coincidence problem, some sophisticated dynamical scalar field DE models, such as quintessence, k-essence and phantom field, have been put forth to replace the cosmological constant [5]. However, these scalar field DE models can not still resolve the coincidence problem.

The interacting DE model has been regarded as a possible way to alleviate this problem [6]. In these models it is assumed that there exists a nonzero interaction between DE and DM in the Universe that gauges DE transfers to DM which allows us to create an equilibrium balance in the evolution of the Universe, so that the density of DE keeps the same order as that of DM at late times. Although lacking of a fundamental explanation for the source of the interaction, the interacting models are manifested as the useful and robust models at the order of one standard deviation by some data sets from observational cosmology including CMB shift parameter, BAO, age parameter and supernova observations and so on 6, 7, 8|. Therefore, the interacting models have attracted a great deal of interest [9, 10, 11, 12, 13, 14, 15, 16, 17, 18, 19, 20, 21, 22,

23, 24, 25]. Recently, some attempts have been proposed to describe the interaction between DE and DM from a fundamental field theory point of view [26, 27].

The investigations above have been focused only on the interaction between DE and DM. However, it is physically reasonable and even expected from a theoretical point of view, that DM as well as DE can interact with other dark components of the universe [28, 29]. Recent investigations shows that unparticle can be treated theoretically as another important dark components in the Universe. The concept of unparticle is introduced first by Georgi [30], which is based on the hypothesis that there could be an exact scale invariant hidden sector resisted at a high energy scale (for a recent review of unparticles, see [33, 34]). Although the 
fundamental energy scale of such a sector is far beyond the reach of todays or near future accelerators, it is possible that this new sector affects low energy phenomenology. These effects are described as unparticle in the effective low energy field theory because that the behaviors of these new degrees of freedom are quite a different those of the ordinary particles. For example, their scaling dimension does not have to be an integer or half an integer. Recently, a lot of work [31, 32] have been focused on the new collider signals for unparticle physics. One of interesting feature of unparticle is that it does not have a definite mass and instead has a continuous spectral density as a consequence of scale invariance [30]

$$
\rho\left(P^{2}\right)=A_{d_{u}} \theta\left(P^{0}\right) \theta\left(P^{2}\right)\left(P^{2}\right)^{d_{u}-2}
$$

where $P$ is the 4 -momentum, $A_{d_{u}}$ is the normalization factor and $d_{u}$ is the scaling dimension. The theoretical bounds of the scaling dimension $d_{u}$ are $1 \leq d_{u} \leq 2$ (for boson unparticle) or $3 / 2 \leq d_{u} \leq 5 / 2$ (for fermion unparticle) [34]. The pressure and energy density of the thermal boson unparticle are given by [35]

$$
\begin{aligned}
& p_{u}=g_{s} T^{4}\left(\frac{T}{\Lambda_{u}}\right)^{2\left(d_{u}-1\right)} \frac{\mathcal{C}\left(d_{u}\right)}{4 \pi^{2}}, \\
& \rho_{u}=\left(2 d_{u}+1\right) g_{s} T^{4}\left(\frac{T}{\Lambda_{u}}\right)^{2\left(d_{u}-1\right)} \frac{\mathcal{C}\left(d_{u}\right)}{4 \pi^{2}}
\end{aligned}
$$

where $\mathcal{C}\left(d_{u}\right)=B\left(3 / 2, d_{u}\right) \Gamma(2 d u+2) \zeta(2 d u+2)$, while $B, \Gamma, \zeta$ are the Beta, Gamma and Zeta functions, respectively. Thus, the EoS of boson unparticle reads [35]

$$
\omega_{u}=\frac{1}{2 d_{u}+1}
$$

For the fermion unparticle, we find the EoS has the same form as that of boson one. Obviously, the EoS of unparticle $\omega_{u}$ is positive which is different from that of DE and DM. Since the unparticle interacts weakly with standard model particles, it can be regarded as a new form of dark component. Recent investigations show that the unparticles play an important role in the early universe [36] and black hole physics [37]. Therefore, it is natural to ask whether there exists some new properties in the late-time evolution of the Universe if the unparticle takes part in the interaction with DE and DM? In this paper we choose four different coupling terms and study the dynamical behaviors of the interacting DE models with DM and unparticle by the phase space analysis method to discuss further stability of the critical points and their cosmological implications.

The paper is organized as follows: in sections II, we construct a cosmological scenario in which DE interacts with DM and unparticle, and then we present the formalism for its transformation into an autonomous dynamical system which is suitable for a phase space stability analysis. In Sec.III, we consider some special 
coupling forms and perform the phase space analysis of the corresponding interacting DE model with DM and unparticle, and then discuss their cosmological implications. Our conclusions and discussions will be presented in the last section.

\section{INTERACTING DARK ENERGY MODEL WITH DARK MATTER AND UNPARTICLE}

In the Einstein theory, the a flat FRW universe is described by the standard Friedmann equation and Raychaudhuri field equation

$$
\begin{aligned}
& H^{2}=\frac{\kappa}{3} \rho, \\
& \dot{H}=-\frac{\kappa}{2}(\rho+p) .
\end{aligned}
$$

$H$ is the Hubble parameter and $\kappa$ is the constant $8 \pi G$. The total energy density $\rho=\rho_{m}+\rho_{x}+\rho_{u}$, where $\rho_{m}$, $\rho_{x}$ and $\rho_{u}$ correspond to the energy densities of DM, DE and unparticle, respectively. For simplicity here we have neglected the radiation and baryons since we are concentrating on the late time accelerating Universe.

The interaction among DE, DM and unparticle can be described in the background by the balance equations

$$
\begin{aligned}
& \dot{\rho_{x}}+3 H\left(1+\omega_{x}\right) \rho_{x}=\Gamma_{1}, \\
& \dot{\rho_{m}}+3 H \rho_{m}=\Gamma_{2}, \\
& \dot{\rho_{u}}+3 H\left(1+\omega_{u}\right) \rho_{u}=\Gamma_{3}
\end{aligned}
$$

Here the terms $\Gamma_{1}, \Gamma_{2}$ and $\Gamma_{3}$ describe the coupling among DE, DM and unparticles. The total conservation equation demands that

$$
\Gamma_{1}+\Gamma_{2}+\Gamma_{3}=0
$$

To analyze the evolution of the dynamical system, we introduce the dimensionless variables

$$
x \equiv \frac{\kappa \rho_{x}}{3 H^{2}}, \quad y \equiv \frac{\kappa \rho_{m}}{3 H^{2}}, \quad z \equiv \frac{\kappa \rho_{u}}{3 H^{2}}, \quad \frac{d}{d N}=\frac{1}{H} \frac{d}{d t},
$$

where $N \equiv \ln a$ is the number of $e$-folding to represent the cosmological time. Using the above definitions, the Hubble equations can be rewritten as

$$
x+y+z=1,
$$

and

$$
\frac{\dot{H}}{H^{2}}=-\frac{3}{2}\left[1+\frac{\omega_{x} x+\omega_{u} z}{x+y+z}\right]=-\frac{3}{2}\left(1+\omega_{x} x+\omega_{u} z\right)
$$


The effective total EOS $\omega_{t o t}$ is given by

$$
\omega_{t o t}=\frac{\omega_{x} \rho_{x}+\omega_{u} \rho_{u}}{\rho_{x}+\rho_{m}+\rho_{u}}=\frac{\omega_{x} x+\omega_{u} z}{x+y+z}=\omega_{x} x+\omega_{u} z
$$

Once the concrete forms of the coupling terms $\Gamma_{1}, \Gamma_{2}$ and $\Gamma_{3}$ are given, the equations of motion (44), (15) and (6) can be transformed to an autonomous system containing the variables $x$ and $y$ and their derivatives with respect to $N=\ln a$. This autonomous system has generally the form: $X^{\prime}=f(X)$, where $X$ is the column vector constituted by the auxiliary variables, $f(X)$ is the corresponding column vector of the autonomous equations, and prime denotes derivative with respect to $N=\ln a$. Solving the equations $X^{\prime}=0$, we can obtain the critical points $X_{c}$. Then, in order to describe the stability properties of these critical points, we can expand the equations $X^{\prime}=f(X)$ around $X_{c}$ by setting $X=X_{c}+\delta U$ with $\delta U$ the perturbations of the variables considered as a column vector. Thus, for each critical point we can expand the equations for the perturbations up to the first order as: $\delta U^{\prime}=\Xi \cdot \delta U$, where the matrix $\Xi$ contains the coefficients of the perturbation equations. Through the analysis of the eigenvalues of $\Xi$, we can describe the stability of each critical point. In general, for an arbitrary coupling terms $\Gamma_{i}$ it is difficult to obtain the analytical forms of the critical points. Here we only consider some specific forms of $\Gamma_{i}$ and examine the stability of interacting DE models with DM and unparticle in the next section.

\section{ANALYSIS OF STABILITY IN THE PHASE SPACE}

In this section, we will consider some concrete coupling forms $\Gamma_{i}$ and analysis the stability of the corresponding interacting DE models with DM and unparticle, and then discuss their cosmological implications.

\section{A. Interacting model I}

In general, there is as yet no basis in fundamental theory for a specific coupling in the dark sectors because that the nature of dark sectors remain unknown. Thus, all coupling models discussed at the present moment are necessarily phenomenological [6]. There are two criterions to determine whether some models can be more physical justification than the others. One is to confront observations. The other is to examine whether the coupling can lead to accelerated scaling attractor solutions[15], which is a decisive way to achieve similar energy densities in dark sectors and alleviate the coincidence problem. Motivated by analogy with dissipation of cosmological fluids, the coupling terms $\Gamma_{i}$ which are proportioned to the densities $\rho_{x}$ and $\rho_{m}$ have been studied in the context of quintessence [6] and phantom [13, 16, 17, 22] models. In this section, we first 
consider the case in which all of the coupling terms $\Gamma_{i}$ are proportioned to the density of DE $\rho_{x}$. Here we choose $\Gamma_{2}=\Gamma_{3}$ so that we can obtain the analytical expression for the critical point, which is very convenient for us to study the dynamics of the model in the following calculations. The concrete expressions of $\Gamma_{i}$ are

$$
\Gamma_{1}=-6 b H \rho_{x}, \quad \Gamma_{2}=\Gamma_{3}=3 b H \rho_{x},
$$

where $b$ is a positive coupling constant. It means that in this model only DE can convert to DM and unparticle. Using dimensionless variables, the dynamical equations of the system can be expressed as

$$
\begin{aligned}
& x^{\prime}=-6 b x-3 \omega_{x} x+3 x\left(\omega_{x} x+\omega_{u} z\right), \\
& y^{\prime}=3 b x+3 y\left(\omega_{x} x+\omega_{u} z\right), \\
& z^{\prime}=3 b x-3 \omega_{u} z+3 z\left(\omega_{x} x+\omega_{u} z\right) .
\end{aligned}
$$

Solving the equations $x^{\prime}=0, y^{\prime}=0$ and $z^{\prime}=0$, we obtain the critical points $\left(x_{c}, y_{c}, z_{c}\right)$ :

- Point $A_{1}:(0,1,0)$,

- Point $B_{1}:(0,0,1)$,

$$
\text { . Point } C_{1}:\left(\frac{\left(2 b+\omega_{x}\right)\left(2 b+\omega_{x}-\omega_{u}\right)}{\left(\omega_{x}-\omega_{u}\right)\left(b+\omega_{x}\right)+b \omega_{x}}, \frac{-b\left(2 b+\omega_{x}-\omega_{u}\right)}{\left(\omega_{x}-\omega_{u}\right)\left(b+\omega_{x}\right)+b \omega_{x}}, \frac{-b\left(2 b+\omega_{x}\right)}{\left(\omega_{x}-\omega_{u}\right)\left(b+\omega_{x}\right)+b \omega_{x}}\right) \text {. }
$$

The points $A_{1}$ and $B_{1}$ imply that our Universe is dominated by DM and unparticle, respectively. The condition for the existence of the point $C_{1}$ is $0<b<-\frac{\omega_{x}}{2}$. The critical point $C_{1}$ denotes that the DE, DM and unparticle can be coexisted in the late-times of the Universe.

After some operations, we can obtain the $3 \times 3$ matrix $\Xi$ of the linearized perturbation equations

$$
\Xi=\left[\begin{array}{ccc}
\omega_{x}\left(6 x_{c}-3\right)+3 \omega_{u} z_{c}-6 b & 0 & 3 \omega_{u} x_{c} \\
3\left(\omega_{x} y_{c}+b\right) & 3\left(\omega_{x} x_{c}+\omega_{u} z_{c}\right) & 3 \omega_{u} y_{c} \\
3\left(\omega_{x} z_{c}+b\right) & 0 & 3 \omega_{x} x_{c}+\omega_{u}\left(6 z_{c}-3\right)
\end{array}\right] .
$$

The eigenvalues of the coefficient matrix $\Xi$ encode the behavior of the dynamical system near the critical points. If the real parts of all of the eigenvalues of the matrix $\Xi$ are negative, then the critical point is a stable point, otherwise it is unstable. Through some careful calculations, we obtain the eigenvalues of the coefficient matrix for these critical points

$$
\begin{array}{ll}
\text { Point } A_{1}: & \lambda_{1}=0, \quad \lambda_{2}=-3 \omega_{u}, \quad \lambda_{3}=-3\left(2 b+\omega_{x}\right), \\
\text { Point } B_{1}: & \lambda_{1}=3 \omega_{u}, \quad \lambda_{2}=3 \omega_{u}, \quad \lambda_{3}=-3\left(2 b+\omega_{x}-\omega_{u}\right), \\
\text { Point } C_{1}: & \lambda_{1}=3\left(2 b+\omega_{x}\right), \quad \lambda_{2}=3\left(2 b+\omega_{x}\right), \quad \lambda_{3}=3\left(2 b+\omega_{x}-\omega_{u}\right) .
\end{array}
$$


For the point $A_{1}$, the eigenvalue $\lambda_{1}$ is non-negative, which indicates that $A_{1}$ is not a stable point. For the point $B_{1}$, we also find that both of the eigenvalues $\lambda_{1}$ and $\lambda_{2}$ are positive because that the EoS of the unparticle $\omega_{u}>0$. Therefore the point $B_{1}$ is not the stable point. For the point $C_{1}$, when $0<b<-\frac{\omega_{x}}{2}$, all of the eigenvalues $\lambda_{1}, \lambda_{2}$ and $\lambda_{3}$ are negative, which means that $C_{1}$ is a stable point as shown in figure (1). The stable region of $C_{1}$ is not affected by the EoS of the unparticle, but the position of $C_{1}$ in the phase space is decided together by $\omega_{x}, \omega_{u}$ and the coupling constant $b$. From Eq.(11), we also learn that the effective total EoS at point $C_{1}$ is $\omega_{t o t}=2 b+\omega_{x}$, which is independent of $\omega_{u}$. When $b \rightarrow-\omega_{x} / 2$, we find that $\omega_{t o t}$ tends to zero. It is consistent with the result shown in figure (2) in which as the coupling is strong enough the Universe will be dominated by DM. Moreover, for the critical point $C_{1}$, we find that in its stable region none of the coordinates $x_{c}, y_{c}$ and $z_{c}$ in phase space vanishes, which means that the coincidence problem can be alleviated in the Universe described by the point $C_{1}$. Since the effective total EoS at point $C_{1}$ is $\omega_{t o t}=2 b+\omega_{x}$ we else obtain $\ddot{a} \propto-\left(3 \omega_{x}+6 b+1\right) t^{\frac{2}{3\left(\omega_{x}+2 b+1\right)}}-2$ and $\rho \propto a^{-3\left(\omega_{x}+2 b+1\right)}$. It means that point $C_{1}$ is an accelerated scaling solution as $b<-\frac{\omega_{x}}{2}-\frac{2}{3}$ and there is singularity in the finite future as $b<\frac{\omega_{x}}{2}-\frac{1}{2}$.

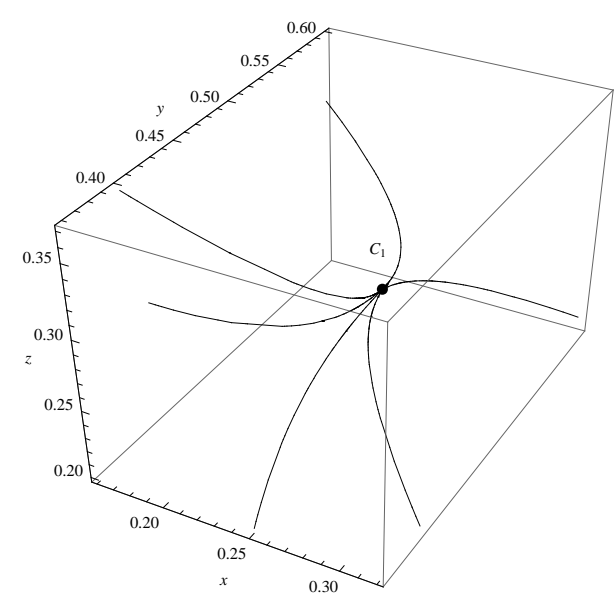

FIG. 1: The phase diagram of interacting dark energy with DM and unparticle through the coupling terms (12). The point $C_{1}$ is the critical point. Here we choose the values $\omega_{x}=-1.2, \omega_{u}=0.28$ and $b=0.5$ in the stable region $b<-\omega_{x} / 2$.

\section{B. Interacting model II}

In the previous discussions, we studied only the case DE converts to DM and unparticle and do not consider the exchange of energy between DM and unparticle. In this section, we will consider another special case in which besides DE transfers to DM, DM can also be converted to unparticle. Similarly, in order to obtain the 


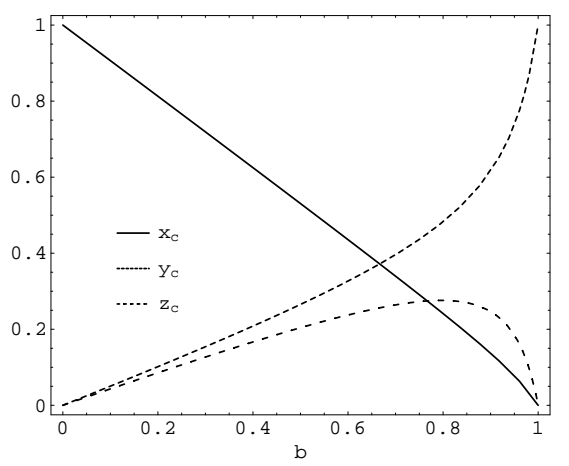

FIG. 2: Variety of $x_{c}, y_{c}$ and $z_{c}$ with $b$ at the critical point $C_{1}$ for fixed $\omega_{x}=-2$ and $\omega_{u}=0.3$. The coupling constant $b$ is located in the stable region $0<b<-\omega_{x} / 2$.

analytical forms for the critical points, we assume that the coupling terms $\Gamma_{i}$ have the forms

$$
\Gamma_{1}=-3 b H \rho_{x}, \quad \Gamma_{2}=3 b H\left(\rho_{x}-\rho_{m}\right), \quad \Gamma_{3}=3 b H \rho_{m},
$$

respectively.

The dynamics of the system can be described by

$$
\begin{aligned}
& x^{\prime}=-3 b x-3 \omega_{x} x+3 x\left(\omega_{x} x+\omega_{u} z\right), \\
& y^{\prime}=3 b(x-y)+3 y\left(\omega_{x} x+\omega_{u} z\right), \\
& z^{\prime}=3 b y-3 \omega_{u} z+3 z\left(\omega_{x} x+\omega_{u} z\right),
\end{aligned}
$$

and the critical points $\left(x_{c}, y_{c}, z_{c}\right)$ are

- Point $A_{2}:(0,0,1)$,

. Point $B_{2}:\left(0,1-\frac{b}{\omega_{u}}, \frac{b}{\omega_{u}}\right)$,

Point $C_{2}:\left(\frac{\omega_{x}\left(b+\omega_{x}-\omega_{u}\right)}{\omega_{x}^{2}+\omega_{u}\left(b-\omega_{x}\right)}, \frac{-b\left(b+\omega_{x}-\omega_{u}\right)}{\omega_{x}^{2}+\omega_{u}\left(b-\omega_{x}\right)}, \frac{b^{2}}{\omega_{x}^{2}+\omega_{u}\left(b-\omega_{x}\right)}\right)$.

The condition that the point $C_{2}$ exists is $0<b<\omega_{u}-\omega_{x}$. Similarly, the $3 \times 3$ matrix $\Xi$ of the linearized perturbation equations reads

$$
\Xi=\left[\begin{array}{ccc}
-3\left(\omega_{x}+b-2 \omega_{x} x_{c}-\omega_{u} z_{c}\right) & 0 & 3 \omega_{u} x_{c} \\
3 \omega_{x}\left(y_{c}+b\right) & 3\left(\omega_{x} x_{c}+\omega_{u} z_{c}\right)-3 b & 3 \omega_{u} y_{c} \\
3 \omega_{x} z_{c} & 3 b & 3 \omega_{x} x_{c}+\omega_{u}\left(6 z_{c}-3\right)
\end{array}\right]
$$

The eigenvalues of the coefficient matrix for these critical points are

$$
\begin{array}{lll}
\text { Point } A_{2}: & \lambda_{1}=3 \omega_{u}, \quad \lambda_{2}=3\left(\omega_{u}-b\right), \quad \lambda_{3}=-3\left(b+\omega_{x}-\omega_{u}\right), \\
\text { Point } B_{2}: & \lambda_{1}=3 b, \quad \lambda_{2}=-3 \omega_{x}, \quad \lambda_{3}=3\left(b-\omega_{u}\right), \\
\text { Point } C_{2}: & \lambda_{1}=3 \omega_{x}, \quad \lambda_{2}=3\left(\omega_{x}+b\right), \quad \lambda_{3}=3\left(b+\omega_{x}-\omega_{u}\right) .
\end{array}
$$


For point $A_{2}$, since $\omega_{u}>0$, the eigenvalue $\lambda_{1}$ always is positive, which indicates that $A_{2}$ is an unstable point. Moreover, the EoS of DE $\omega_{x}<0$ and the coupling constant $b>0$ means that both of the eigenvalues $\lambda_{1}$ and $\lambda_{2}$ of the point $B_{2}$ are positive. Thus the point $B_{2}$ is an unstable point. For the point $C_{2}$, when $-\omega_{x}<b<\omega_{u}-\omega_{x}$, the sign of $\lambda_{2}$ is always opposite to the signs of $\lambda_{1}$ and $\lambda_{2}$, which leads $C_{2}$ to a saddle point. However, when $0<b<-\omega_{x}$, we find that all of eigenvalues $\left(\lambda_{1}, \lambda_{2}\right.$ and $\left.\lambda_{3}\right)$ are negative, which indicates that $C_{2}$ is a stable point as shown in figure (3). Meanwhile, we also find none of the coordinate components of $C_{2}$ in the phase space disappears, which means that in this case there exist three components (DE, DM and unparticle) in the late-time universe and the coincidence problem can be alleviated. From Eq.(11), we learn that the effective total EoS at point $C_{2}$ is $\omega_{t o t}=b+\omega_{x}$. Therefore we obtain $\ddot{a} \propto-\left(1+3 b+3 \omega_{x}\right) t^{\frac{2}{3\left(1+b+\omega_{x}\right)}}-2$ and $\rho \propto a^{-3\left(1+b+\omega_{x}\right)}$. It means that point $C_{2}$ is an accelerated scaling solution as $b<-\omega_{x}-1 / 3$ and there exists singularity in the finite future as $b<-\omega_{x}-1$. For fixed $\omega_{x}$, the effective total EoS increases with $b$. Figure (4) shows that in the Universe described by $C_{2}$ the density of the unparticle increases and DE decreases with the coupling constant $b$, while the density of DM first increases and then decreases. This means that the Universe will be dominated by unparticle if the coupling constant $b$ is large enough.

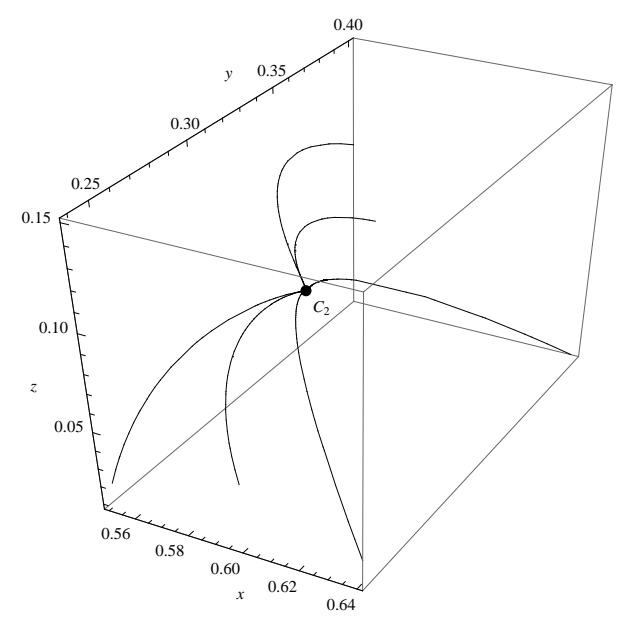

FIG. 3: The phase diagram of interacting dark energy with DM and unparticle through the coupling terms (17). The point $C_{2}$ is the critical point. Here we choose the values $\omega_{x}=-1.2, \omega_{u}=0.28$ and $b=0.5$ in the stable region $b<-\omega_{x}$.

\section{Interacting model III}

In Ref.[21], we have investigated the dynamics of the interacting DE models in which the coupling terms $\Gamma_{i}$ contains the product of the densities of DE and DM and find that this new type of dark sector coupling leads to the more interesting accelerated scaling solutions and presents us more complicated features in dynamical 


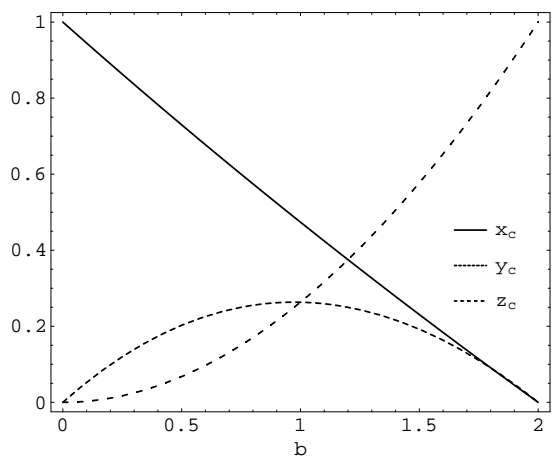

FIG. 4: Variety of $x_{c}, y_{c}$ and $z_{c}$ with $b$ at the critical point $C_{2}$ for fixed $\omega_{x}=-1.8$ and $\omega_{u}=0.2$.

phase space. Therefore, in the following sections, we will consider the cases in which the coupling terms $\Gamma_{i}$ contain the product of the density of DE, DM and unparticle and to see whether it presents some new properties or not in the evolution of the Universe with the unparticle component. For mathematical simplicity, we assume firstly the interaction among DE, DM and unparticle have the forms

$$
\Gamma_{1}=-6 b \kappa H^{-1} \rho_{x} \rho_{u}, \quad \Gamma_{2}=\Gamma_{3}=3 b \kappa H^{-1} \rho_{x} \rho_{u}
$$

As in the model (12), these coupling terms also denote that DE can be transfer to DM and unparticle. The dynamical equations of the system can be written as

$$
\begin{aligned}
& x^{\prime}=-6 b x z-3 \omega_{x} x+3 x\left(\omega_{x} x+\omega_{u} z\right), \\
& y^{\prime}=3 b x z+3 y\left(\omega_{x} x+\omega_{u} z\right), \\
& z^{\prime}=3 b x z-3 \omega_{u} z+3 z\left(\omega_{x} x+\omega_{u} z\right),
\end{aligned}
$$

and the critical points $\left(x_{c}, y_{c}, z_{c}\right)$ are

- Point $A_{3}:(1,0,0)$,

- Point $B_{3}:(0,0,1)$,

- Point $C_{3}:\left(\frac{\omega_{u}\left(2 b+\omega_{x}-\omega_{u}\right)}{b\left(2 b+2 \omega_{x}-\omega_{u}\right)}, \frac{\left(\omega_{x}-\omega_{u}\right)^{2}+3 b\left(\omega_{x}-\omega_{u}\right)+2 b^{2}}{b\left(2 b+2 \omega_{x}-\omega_{u}\right)},-\frac{b\left(b+\omega_{x}-\omega_{u}\right)}{b\left(2 b+2 \omega_{x}-\omega_{u}\right)}\right)$.

The condition that the point $C_{3}$ exists is $b>\omega_{u}-\omega_{x}$. Repeating the previous operations, we can obtain the $3 \times 3$ matrix $\Xi$ of the linearized perturbation equations

$$
\Xi=\left[\begin{array}{ccc}
\omega_{x}\left(6 x_{c}-3\right)+3 \omega_{u} z_{c}-6 b & 0 & 3 \omega_{u} x_{c}-6 b \\
3\left(\omega_{x} y_{c}+b z_{c}\right) & 3\left(\omega_{x} x_{c}+\omega_{u} z_{c}\right) & 3\left(\omega_{u} y_{c}+b x_{c}\right) \\
3\left(\omega_{x}+b\right) z_{c} & 0 & 3 \omega_{x} x_{c}+\omega_{u}\left(6 z_{c}-3\right)+3 b
\end{array}\right]
$$


and the eigenvalues of the coefficient matrix for these critical points

$$
\begin{array}{ll}
\text { Point } A_{3}: & \lambda_{1}=3 \omega_{x}, \quad \lambda_{2}=3 \omega_{x}, \quad \lambda_{3}=3\left(b+\omega_{x}-\omega_{u}\right), \\
\text {. Point } B_{3}: & \lambda_{1}=3 \omega_{u}, \quad \lambda_{2}=3 \omega_{u}, \quad \lambda_{3}=-3\left(2 b+\omega_{x}-\omega_{u}\right), \\
\text {. Point } C_{3}: & \lambda_{1}=\frac{3 \omega_{u} \omega_{x}}{2 b+2 \omega_{x}-\omega_{u}}, \\
\lambda_{2,3}=\frac{3\left(b \omega_{u} \omega_{x} \pm \sqrt{\left(b \omega_{u} \omega_{x}\left[16 b^{3}+8 b^{2}\left(5 \omega_{x}-4 \omega_{u}\right)+4\left(\omega_{x}-\omega_{u}\right)^{2}\left(2 \omega_{x}-\omega_{u}\right)+b\left(32 \omega_{x}^{2}-51 \omega_{x} \omega_{u}+20 \omega_{u}^{2}\right)\right]\right.}\right)}{2 b\left(2 b+2 \omega_{x}-\omega_{u}\right)} .
\end{array}
$$

Through the similar analysis, we find that $A_{3}$ is a stable point when $b<\omega_{u}-\omega_{x}$ and is a saddle point
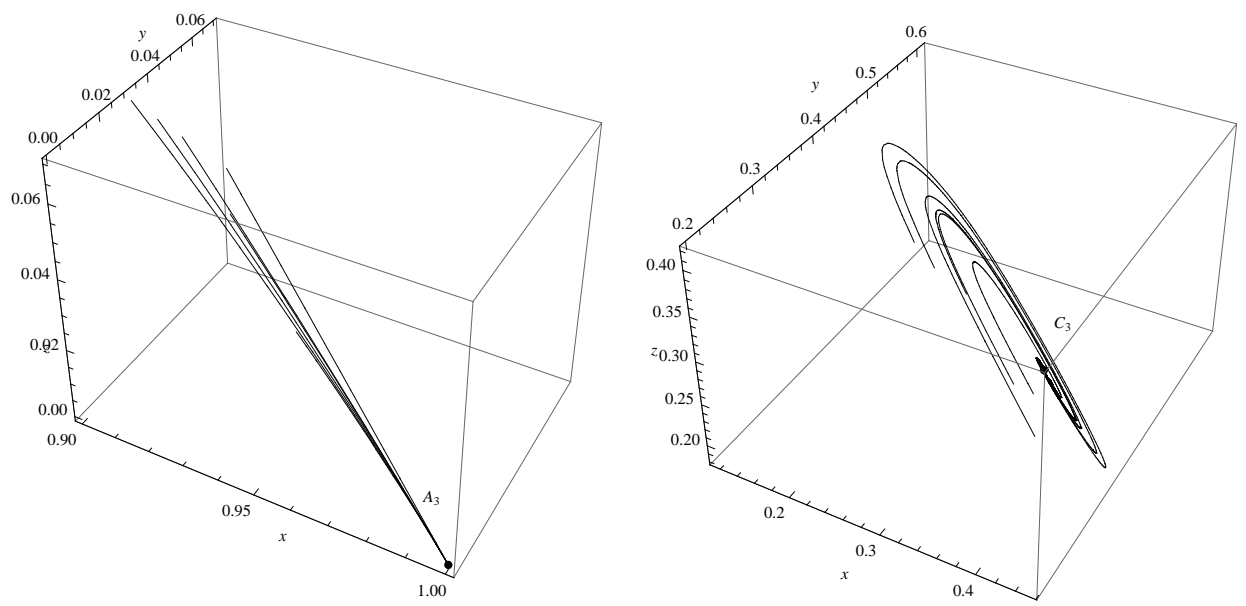

FIG. 5: The phase diagram of interacting dark energy with DM and unparticle through the coupling terms (22). In the left figure, we fix the values $\left(\omega_{x}=-1.2, \omega_{u}=0.28\right.$ and $\left.b=0.5\right)$ which meets $b<\omega_{u}-\omega_{x}$ and the critical point is $A_{3}$. In the right, we choose the values $\left(\omega_{x}=-1.2, \omega_{u}=0.28\right.$ and $\left.b=1.8\right)$ which satisfies $b>\omega_{u}-\omega_{x}$ and the critical point is $C_{3}$.

when $b>\omega_{u}-\omega_{x}$. The point $B_{3}$ is an unstable point because that $\omega_{u}>0$, which leads to the signs of the eigenvalues $\lambda_{1,2}$ always are positive. For the point $C_{3}$, it is a stable point when $b>\omega_{x}-\omega_{u}$. We can obtain the total effective $\operatorname{EoS} \omega_{t o t}=\frac{\omega_{u} \omega_{x}}{2 b+2 \omega_{x}-\omega_{u}}$. It depends not only on the EoS of DE and unparticle, but also on the coupling constant $b$. From the total effective $\operatorname{EoS} \omega_{t o t}$, it is easy to obtain that point $C_{3}$ is an accelerated scaling solution as $\omega_{u}-\omega_{x}<b<\left(\omega_{u}-2 \omega_{x}-3 \omega_{x} \omega_{u}\right) / 2$. While for $b>\left(\omega_{u}-2 \omega_{x}-3 \omega_{x} \omega_{u}\right) / 2$, we find that $\omega_{t o t}>-1 / 3$, which means that it is a decelerated scaling solution. This can be explained by that the larger $b$ denote the more DE transfers to DM and unparticle in the Universe. While, as $b<\omega_{x}-\omega_{u}$, the point $C_{3}$ is an unstable point. Moreover, in this model, when $b$ is small the coupling the Universe will enter the era dominated by DE because $A_{3}$ is a stable point (as shown in the left figure in Fig.(5)). When $b$ is larger the Universe will enter a stable stage described by point $C_{3}$ (as shown in the right figure in Fig.(5)), 
which contains the DE, DM and unparticle. Thus, when $b>\omega_{u}-\omega_{x}$ this model can resolve the coincidence problem. Moreover, from the Fig.(66), we also find with the increase of $b$ the density of DM increases in the Universe, but both of DE and unparticle decrease. This implies that the Universe will be dominated by DM when the interaction is very strong. Figure (7) also tells us that with the increase of the coupling constant $b$ the EoS $\omega_{t o t}$ increases so that the Universe can make a transition from an accelerating expansion phase to a decelerating one. As $b$ tends to infinity the EoS $\omega_{t o t}$ approaches to zero, which is consistent with that of DM.

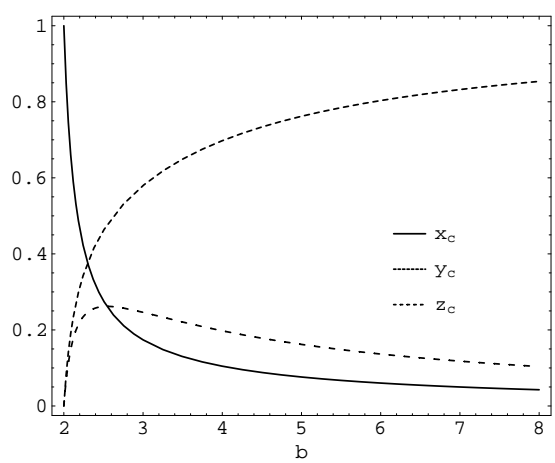

FIG. 6: Variety of $x_{c}, y_{c}$ and $z_{c}$ with $b$ at the critical point $C_{3}$ for fixed $\omega_{x}=-1.7$ and $\omega_{u}=0.3$. Here the coupling constant $b$ is located in the region $b>\omega_{u}-\omega_{x}$.

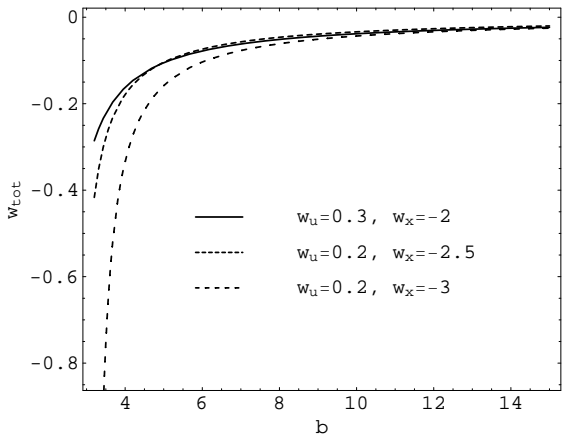

FIG. 7: Variety of the effective EoS $\omega_{\text {tot }}$ with $b$ at the critical point $C_{3}$ for different $\omega_{x}$ and $\omega_{u}$. Here the coupling constant $b$ is limited in the region $b>\omega_{u}-\omega_{x}$.

\section{Interacting model IV}

In the model (III), we consider only the case the coupling terms do not contain the density of DM. In the following model, we will consider the interaction includes $\rho_{m}$ and suppose that $\Gamma_{i}$ have the forms

$$
\Gamma_{1}=-3 b \kappa H^{-1} \rho_{x} \rho_{u}, \quad \Gamma_{2}=3 b \kappa H^{-1}\left(\rho_{x} \rho_{u}-\rho_{m} \rho_{u}\right), \quad \Gamma_{3}=3 b \kappa H^{-1} \rho_{m} \rho_{u} .
$$


Although the coupling forms are more complicated than the model (22), it also presents us the analytical expressions for the critical points which brings us the simplicity in the later calculation. This type of $\Gamma_{i}$ also denote DE can be transfer to DM and at the same time DM can also be convert to unparticle. The dynamical equations of the system are given by

$$
\begin{aligned}
& x^{\prime}=-3 b x z-3 \omega_{x} x+3 x\left(\omega_{x} x+\omega_{u} z\right), \\
& y^{\prime}=3 b(x z-y z)+3 y\left(\omega_{x} x+\omega_{u} z\right), \\
& z^{\prime}=3 b y z-3 \omega_{u} z+3 z\left(\omega_{x} x+\omega_{u} z\right),
\end{aligned}
$$

and the critical points $\left(x_{c}, y_{c}, z_{c}\right)$ can be expressed as

$$
\begin{aligned}
& \text { - Point } A_{4} \text { : }(1,0,0) \text {, } \\
& \text { - Point } B_{4} \text { : }(0,0,1) \text {, } \\
& \text {. Point } C_{4}:\left(\frac{b+\omega_{x}-\omega_{u}}{b}, \frac{\left(\omega_{u}-\omega_{x}\right)\left(b+\omega_{x}-\omega_{u}\right)}{b\left(b-\omega_{u}\right)},-\frac{\omega_{x}\left(\omega_{x}-\omega_{u}\right)}{b\left(b-\omega_{u}\right)}\right) \text {. }
\end{aligned}
$$

The critical point $C_{4}$ exists only when $b>\omega_{u}-\omega_{x}$. Similarly, we can obtain the $3 \times 3$ matrix $\Xi$

$$
\Xi=\left[\begin{array}{ccc}
\omega_{x}\left(6 x_{c}-3\right)+3 \omega_{u} z_{c}-3 b & 0 & 3\left(\omega_{u}-b\right) x_{c} \\
3\left(\omega_{x} y_{c}+b z_{c}\right) & 3\left(\omega_{x} x_{c}+\omega_{u} z_{c}-b z_{c}\right) & 3\left(\omega_{u} y_{c}+b x_{c}-b y_{c}\right) \\
3 \omega_{x} z_{c} & 3 b z_{c} & 3 \omega_{x} x_{c}+\omega_{u}\left(6 z_{c}-3\right)+3 b y_{c}
\end{array}\right]
$$

The eigenvalues of the coefficient matrix for these critical points are

$$
\begin{array}{ll}
\text { Point } A_{4}: & \lambda_{1}=3 \omega_{x}, \quad \lambda_{2}=3 \omega_{x}, \quad \lambda_{3}=3\left(\omega_{x}-\omega_{u}\right) \\
\text { Point } B_{4}: & \lambda_{1}=3 \omega_{u}, \quad \lambda_{2}=3\left(\omega_{u}-b\right), \quad \lambda_{3}=-3\left(b+\omega_{x}-\omega_{u}\right) \\
\text { Point } C_{4}: & \lambda_{1}=\frac{3 \omega_{x}\left(b+\omega_{x}-2 \omega_{u}\right)}{b-\omega_{u}}, \quad \lambda_{2,3}=\frac{3\left(b \omega_{x} \pm \sqrt{b \omega_{x}\left[4\left(\omega_{x}-\omega_{u}\right)^{2}+b\left(5 \omega_{x}-4 \omega_{u}\right)\right]}\right)}{2 b} .
\end{array}
$$

Obviously, all of eigenvalues $\left(\lambda_{1}, \lambda_{2}\right.$ and $\left.\lambda_{3}\right)$ for the point $A_{4}$ are negative because the EoS of DE $\omega_{x}<0$, which indicates that in this case the point $A_{4}$ always is a stable point. The point $B_{4}$ is an unstable point since $\lambda_{1}$ is positive. From the previous discussion we find that the point $C_{4}$ exists only when $b>\omega_{u}-\omega_{x}$, which leads to that $\lambda_{2}>0$ and $\lambda_{3}<0$. This means that the point $C_{4}$ is a saddle point. Since the point $A_{4}$ describes a Universe filled with only DE, the interaction (27) cannot resolve the coincidence problem.

\section{CONCLUSIONS AND DISCUSSIONS}

In this paper we have studied the dynamical behaviors when DE is coupling to DM and unparticle in the standard flat FRW cosmology. We considered four different interacting models and examined the stability of 
critical points. In all the examined models we found that there exist a stable late-time scaling solution which corresponds to an accelerating universe. This feature was expected since DE cosmology has been constructed in order to always satisfy this condition. Moreover, for all the studied cases, we also find the accelerating stable solutions if we choose the appropriated parameters in the models. Except the fourth model, the stable solutions in other models admit that DE coexists with DM and unparticle in the Universe. Our result also implies that in these models the coincidence problem can be alleviated only when the coupling is not strong enough.

\section{Acknowledgments}

This work was partially supported by the National Natural Science Foundation of China under Grant No.10875041; the Scientific Research Fund of Hunan Provincial Education Department Grant No.07B043 and the construct program of key disciplines in Hunan Province. J. L. Jing's work was partially supported by the National Natural Science Foundation of China under Grant No.10675045 and No.10875040; and the Hunan Provincial Natural Science Foundation of China under Grant No.08JJ3010.

[1] Riess A G et al 1998 Astron. J. 1161009

de Bernardis P et al 2000 Nature 404955

Riess A G et al astro-ph/0611572

[2] Perlmutter S et al 1999 Astrophys. J. 517565

Perlmutter S et al 2003 Astrophys. J. 598102

[3] Eisenstein D J et al 2005 Astrophys. J. 633560

[4] Spergel D N et al 2007 Astrophys. J. Suppl. 170377

[5] Padmanabhan T 2003 Phys. Rept. 380235

Peebles P J E and Ratra B 2003 Rev. Mod. Phys. 75559

Sahni V 2004 Lect Notes Phys. 653141

[6] Zimdahl W, Pavón D and Chimento L P 2001 Phys. Lett. B 521133

Chimento L P, Jakubi A S, Pavón D and Zimdahl W 2003 Phys. Rev. D 67083513

Chimento L P and Pavón D 2006 Phys. Rev. D 73063511

del Campo S, Herrera R and Pavón D 2004 Phys. Rev. D 70043540

Pavón D and Zimdahl W 2005 Phys. Lett. B 628206

[7] He J and Wang B 2008 JCAP 0806010

Feng C, Wang B, Abdalla E and Su R K 2008 Phys. Lett. B 665111

He J, Wang B and Abdalla E 2009 Phys. Lett. B 671139 
[8] Wang B et al 2007 Nucl. Phys. B 77869

[9] Olivares G et al 2005 Phys. Rev. D 71063523

Olivares G et al 2006 Phys. Rev. D 74043521

[10] Wang B, Gong Y and Abdalla E 2005 Phys. Lett. B 624141 Wang B et al 2006 Phys. Lett. B 637357

[11] Das S, Corasaniti P S and Khoury J 2006 Phys. Rev. D 73083509

[12] Amendola L and Tocchini-Valentini D 2001 Phys. Rev. D 64043509

Amendola L, Tsujikawa S and Sami M 2006 Phys. Lett. B 632155

Amendola L and Quercellini C 2003 Phys. Rev. D 68023514

Anderson G W and Carroll S M, astro-ph/9711288

[13] Zimdahl W 2005 Int. J. Mod. Phys. D 142319

[14] Feng C, Wang B, Gong Y and Su R K 2007 JCAP 09005

[15] Bohmer C G et al 2008 Phys. Rev. D 78023505

[16] Guo Z K et al 2007 Phys. Rev. D 76023508

Amendola L, Campos G and Rosenfeld R, astro-ph/0610806

[17] Pavón D and Wang B, arXiv: 0712.0565

[18] Hung P Q 2006 Nucl. Phys. B 74755

[19] Jackson B M et al, arXiv: 0901.3272

[20] Chen X M and Gong Y G arXiv: 0811.1698; arXiv: 0812.1117

[21] Chen S B, Wang B and Jing J L 2008 Phys. Rev. D 78123503

[22] Wu P X and Zhang S N 2008 JCAP 06007

[23] Fu X Y, Yu H W and Wu P X 2008 Phys. Rev. D 78063001

[24] Olivares G et al 2008 Phys. Rev. D 77063513

[25] Manera M and Mota D F 2006 Mon. Not. Roy. Astron. Soc. 3711373

Nunes N J and Mota D F 2006 Mon. Not. Roy. Astron. Soc. 368751

[26] Amendola L 2000 Phys. Rev. D 62043511

Bean R et al 2008 Phys. Rev. D 78123514

[27] Micheletti S, Abdalla E and Wang B arXiv: 0902.0318

[28] Cruz N et al 2008 Phys. Lett. B 663338

[29] Cabral G C et al arXiv: 0812.1827

[30] Georgi H 2007 Phys. Rev. Lett. 98221601

Georgi H 2007 Phys. Lett. B 650275

[31] Zhang C X, Huang M Q and Zhong M 2008 Phys. Rev. D 78096006

He X G and Tsai L 2008 JHEP 0806074 (2008)

He X G and Wen C C 2008 Phys. Rev. D 78017301

[32] Kumar M C et al 2008 Phys. Rev. D 77055013

Feng J L, Rajaraman A and Tu H 2008 Phys. Rev. D 77075007

Kikuchi T, Okada N and Takeuchi M 2008 Phys. Rev. D 77094012

[33] Cheung K M, Keung W Y and Yuan T C arXiv: 0809.0995 
[34] Rajaraman A arXiv: 0809.5092

[35] Chen S L, He X G, Hu X P and Liao Y arXiv: 0710.5129

[36] Davoudiasl H 2007 Phys. Rev. Lett. 99141301 arXiv: 0705.3636

Wei H arXiv: 0812.4489

Gong Y and Chen X L 2008 Eur. Phys. J. C 57785

[37] Mureika J R arXiv: 0808.0523

Dai D C and Stojkovic D arXiv: 0812.3396 